DOI : 10.1097/MPG.0000000000001217

\title{
RADIOLOGIC PREDICTORS OF SURGERY IN NEWLY DIAGNOSED PEDIATRIC CROHN'S DISEASE PATIENTS
}

Damien Parker, MD, Boaz Karmazyn, MD*, and Steven J. Steiner, MD

Division of Pediatric Gastroenterology/Hepatology/Nutrition

*Division of Pediatric Radiology

\section{Riley Hospital for Children}

Indiana University School of Medicine

Indianapolis, Indiana

\section{Address Correspondence to:}

Steven J. Steiner, M.D.

Division of Pediatric Gastroenterology/Hepatology/Nutrition

James Whitcomb Riley Hospital for Children

Indiana University School of Medicine

705 Riley Hospital Drive/ ROC 4210

Indianapolis, Indiana 46202-5225

(317) 944-3774-Office

(317) 944-8521-Fax

Email: ssteiner@iupui.edu

This is the author's manuscript of the article published in final edited form as:

Parker, D., Karmazyn, B., \& Steiner, S. J. (2016). Predictors of Surgery in Newly Diagnosed Pediatric Crohn's Disease Patients. Journal of Pediatric Gastroenterology and Nutrition. http://doi.org/10.1097/MPG.0000000000001217 
No funding sources. No conflicts of interest.

Word Count: 2269

Table and Figures: 3

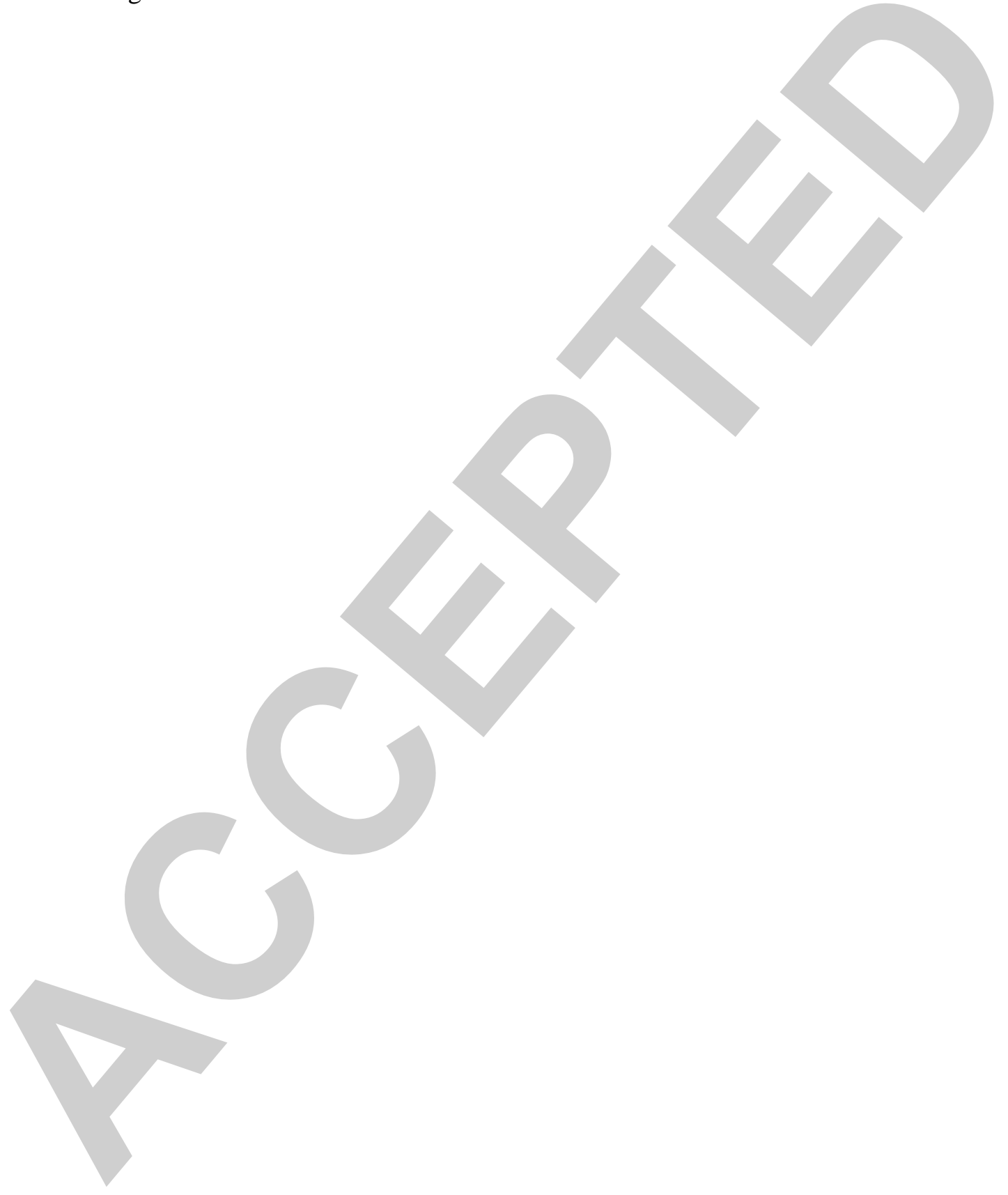

Copyright @ ESPGHAN and NASPGHAN. All rights reserved. 


\begin{abstract}
Objectives: To assess whether small bowel imaging conducted at the time of diagnosis could be used as a predictor of small bowel surgical intervention in a pediatric Crohn’s disease patient population.

Methods: A retrospective analysis of small bowel imaging within 30 days of diagnosis of pediatric Crohn's disease was conducted. Patients were divide into two groups based on small bowel imaging: those with no or minor abnormalities (71\%) and those with more extensive or obstructive abnormalities (29\%). Medical records were reviewed for small bowel surgical intervention and clinic follow-up visits.

Results: 232 patients were included in the study group (average age at diagnosis 11.7 years). Twenty-seven patients (12\%) underwent small bowel surgical intervention. The relative risk for small bowel surgical intervention was 2.91 in the group with more extensive imaging abnormalities. The majority of increased surgical risk occurred in the first year after diagnosis, when the normal-minor group had a $2 \%$ surgical risk and the more abnormal group had a 17\% surgical risk. Both groups had a 2-3\% surgical risk per year after the first year.
\end{abstract}

Conclusions: Small bowel imaging at the time of diagnosis in pediatric Crohn's disease can help predict the risk of small bowel surgical intervention, and should be recommended for all newly diagnosed patients. Nearly one-third of our cohort underwent small bowel surgical intervention through eight years of follow-up. Surgical complications of Crohn's disease often occur in the small bowel, and counseling families about surgical risk is an integral part of pediatric Crohn’s disease management.

Key Words: Crohn disease; pediatrics; small intestine; risk assessment 


\section{What is Known/What is New}

What is Known:

- Pediatric patients with Crohn's disease often require surgical therapy.

- Radiologic evaluation is frequently used to assess Crohn's disease activity and location.

- Multiple clinical factors have been associated with increased risk for surgery.

What is New:

- Significantly abnormal small bowel imaging at diagnosis predicts nearly 3x risk of intestinal surgery.

- Most of this increased risk occurs in the first year after diagnosis.

- Surgery risk remains 2-3\% per year after the first year after diagnosis. 


\section{Introduction}

Over the last decade, advances have occurred in imaging the small bowel in patients with inflammatory bowel disease. In patients with known or suspected Crohn's disease (CD), evaluation of the small bowel may be necessary to assess disease activity and extent, as well as complications such as strictures, abscesses, or fistulas. Recent ESPGHAN (Porto criteria) guidelines include radiological evaluation of the small bowel as a mandatory part of the diagnostic work-up of a newly diagnosed pediatric IBD patient, except in cases where a definitive diagnosis of ulcerative colitis is made(1). Detection of complications, such as irreversible bowel stricture, abscess formation or fistulas, can affect medical or surgical management.

A small bowel follow-through (SBFT) was used for many years as the radiologic test of choice for evaluation of the small bowel. More recently, cross-sectional studies, such as computed tomography enterography (CTE) and magnetic resonance enterography (MRE) are more commonly used for assessing small bowel Crohn's disease(2). The main advantages of cross sectional imaging are improved assessment of disease activity and increased sensitivity in the detection of extramural complications. The choice of imaging modality involves consideration of diagnostic accuracy, cost, radiation exposure, and availability. In addition, novel diagnostic tests, such as capsule endoscopy(3) and balloon enteroscopy $(4,5)$, are being used selectively by pediatric gastroenterologists to assess the small bowel.

The purpose of this study was to assess whether SBFT study conducted at the time of diagnosis could be used as a predictor of small bowel surgical intervention in a pediatric Crohn's disease patient population. We chose to use SBFT in this retrospective study to amass the largest amount of information and follow-up duration as possible. We believe these results can be extrapolated to cross sectional imaging which is becoming standard in CD. Our 
hypothesis was that a greater percentage of patients with abnormal small bowel imaging at the time of diagnosis would require small bowel surgical intervention over time as compared to those with normal small bowel imaging.

\section{Materials and Methods}

This study was approved by the Indiana University Institutional Review Board.

The medical records of pediatric patients with an established diagnosis of Crohn's Disease, treated in the Division of Pediatric Gastroenterology/Hepatology/Nutrition at Riley Children's Hospital between 1994 and 2011, were reviewed. These patients were identified from an IRB approved Pediatric Inflammatory Bowel Disease database currently maintained by the division. The clinical data of the patients obtained included gender, age, date of diagnosis, date of initial diagnostic endoscopy, most recent pediatric GI clinic visit date, and date and type of surgical intervention if performed.

Patients were included if small bowel follow-through imaging was obtained within three months of the date of diagnosis. We created a modified SBFT scoring system based on the grading system of Buchman et al.(6) These radiological studies were graded based on the original clinical description of findings in the official report and available radiological images. These SBFT studies were graded from 0 to 4 using the following scale: Grade 0 (normal), Grade 1 (minimal nodularity, ulcerations, normal luminal diameter, $<5 \mathrm{~cm}$ involved), Grade 2 (more extensive ulcers, minimal luminal narrowing, 5-10 cm involved), Grade 3 (fistula, skip areas, extensive ulceration, $>10 \mathrm{~cm}$ involved but no proximal bowel dilatation) and Grade 4 (proximal bowel dilatation). Patients who had SBFT findings which were interpreted as being normal or mildly abnormal based on the studies grading system (Grades 0-2), were compared to patients with SBFT imaging defined as significantly abnormal (Grades 3-4). The patients' 
medical records were then reviewed to determine if they required surgery related to their Crohn's disease. The surgical interventions documented included any bowel resections, fistula closures, or other small bowel surgeries.

The statistical analysis was based on survival time, which was determined to be the time between the date of diagnosis and date of surgery for the patient, with patients censored at the date of last recorded GI clinic visit. These two groups were compared using KaplanMeier analysis examining surgery free status over time. A chi-square analysis was conducted to determine if the rates of surgical intervention were different between the two groups over the length of the study. A value of $p<0.05$ was considered to represent the presence of a statistically significant difference. We calculated a cumulative surgical frequency by using a Kaplan-Meier survival probability estimate over each time period. Annual surgical risk was determined by dividing the number of patients who underwent surgical intervention by the number of patients still being followed at the beginning of that year. Censored patients were removed from the total surgical-free patients at the end of each year, which gives the number of patients still being followed for the beginning of the next year. Compound conditional probability was then used to calculate the overall surgical risk at the end of each subsequent year. All values are described as mean \pm standard deviation, except where noted.

\section{Results}

The medical records of more than 500 pediatric patients with an established diagnosis of Crohn's Disease were reviewed. Two hundred and forty patients were identified to have a SBFT performed within three months of the time of their diagnosis of Crohn's Disease. One patient was excluded from the study group because no records of pediatric GI clinic visitations were obtained indicating a lack of follow-up. Four patients were excluded from the study group due to difficulty interpreting their SBFT radiological reports due to minimal 
information reported, vague descriptive terminology used, and lack of quality available imaging. Three patients were excluded from the study group because they had surgical intervention prior to their diagnosis which may have been a result of Crohn's disease.

The final study group included 232 patients with a mean age at the time of diagnosis of $11.7 \pm 3.5$ years. The study population consisted of 98 females and 134 males. The average time from diagnosis to the time of SBFT was $7 \pm 17$ days.

Eighty-six patients (37\%) were given a Grade 0 (normal) using the SBFT grading scale (Figure 1). Sixty-seven patients (29\%) were given a Grade 1, thirty patients (13\%) were given a Grade 2, thirty-nine patients (17\%) were given a Grade 3, and ten patients (4\%) were given a Grade 4.

Twenty-seven patients (12\%) were identified who underwent small bowel surgical intervention for Crohn's disease during follow-up. Some patients underwent more than one surgical intervention at the time of their first surgery. Eighteen patients (8\%) underwent ileocecal resection with primary anastomosis. Seven patients (3\%) underwent small bowel resection- six ileal and one jejunal, with primary anastamosis. Two patients underwent fistula closure. One patient underwent gastrojejunostomy for duodenal stricture. The surgical frequency of the entire cohort of patients was $5 \%$ by one year, $16 \%$ by 5 years, and 33\% by 8 years after diagnosis (Table 1$)$.

Sixteen of the twenty-seven patients who underwent surgery were from Grades 0-2, while 11 were from Grades 3-4. Kaplan-Meier analysis (Figure 2) revealed a significantly $(\mathrm{p}<0.01)$ different risk of surgical resection between patients with Grades 0-2 and Grades 3-4. The relative risk of small bowel surgical intervention was 2.91 with standard error of 1.14 for patients with Grades 3-4 compared to patients with Grades 0-2.

The surgical frequency in patients with Grades $0-2$ was $2 \%$ by one year, $11 \%$ by 5 years, and 29\% by 8 years after diagnosis (Table 1). The surgical frequency in patients with 
Grades $3-4$ was $17 \%$ by one year, $27 \%$ by 5 years, and $42 \%$ by 8 years after diagnosis. The difference in surgical intervention between the two groups was nearly completely explained by the different rates of surgical intervention in the first year. Among all patients, after the first year, the frequency of small bowel surgical intervention was 2-3\% per year.

\section{Discussion}

Our study demonstrates that SBFT imaging conducted at the time of diagnosis in pediatric Crohn's disease helps predict the risk of surgical resection. The data obtained from our study suggest that there is a strong correlation between markedly abnormal small bowel imaging at the time of diagnosis and increased future risk of the pediatric patient requiring small bowel surgery. Patients in our study with more extensive SBFT abnormalities at the time of diagnosis had a nearly three-fold increased risk for small bowel surgery. Reliable detection and classification of these abnormalities by the pediatric gastroenterologist would prove to be invaluable in patient counseling and management.

Our patients with more abnormal radiologic studies of small bowel at diagnosis of Crohn's disease had an increased risk of surgery in the first year after diagnosis (2\% vs. 17\%). Delay in diagnosis has been correlated with the risk of stenotic disease and intestinal surgery.(7) Following the first year, the rates of surgery were fairly similar between the two groups, between 2-3\% per year between years 1 and 5 after diagnosis. This may indicate that patients with a fibrostenotic phenotype of Crohn's disease may have significant stenosis at diagnosis which is not amenable to medical therapy, and these patients may benefit from more rapid surgical therapy. As newer therapies become available and widely used in pediatric patients, the risk of surgery in patients with more abnormal small bowel imaging may be 
decreased. Regardless of the initial radiologic findings, current therapies appear to be limiting further stenotic disease and small bowel surgical resection to a low rate.

Previously published longitudinal studies of Crohn's disease patients demonstrate that pediatric and adult Crohn's disease patients have increased risk of surgery over time. Gupta et al. demonstrated that pediatric Crohn’s patients had a cumulative incidence of surgery of $6 \%$ at 1 year, $17 \%$ at 5 years and $28 \%$ at 10 years. This study also demonstrated that development of an abscess, stricture or fistula was associated with an increased risk of surgery.(8) As an overall cohort, the frequency of surgery we observed was remarkably similar to that of Gupta et al. Longitudinal studies in the adult Crohn's population have shown a greater cumulative incidence of surgery over time. Bernell et al.(9) described a cumulative rate of intestinal resection of $44 \%, 62 \%$ and $71 \%$ at 1,5 and 10 years following diagnosis.

Multiple clinical factors have been associated with an increased risk of intestinal surgery in Crohn's disease.(10) Surgical risk may be increased in the presence of a NOD2 mutation. $(11,12)$ The presence of perianal disease was independently associated with the need for surgery.(12) The presence of ASCA Ig positive serology was also associated with stricturing disease and surgery.(13) Interestingly, mood or anxiety co-morbidity was associated with a $28 \%$ increased risk for surgery.(14) In a pediatric cohort, stenotic disease, penetrating disease, upper gastrointestinal involvement, and age at diagnosis of less than 14 years were associated with increased risk of a second surgical resection.(15) In a query of the NIDK IBD Genetics Consortium, the presence of any L4 disease phenotype was associated with stricturing disease, and L4-jejunal patients were more than twice as likely as L4-EGD patients to have had any or multiple surgeries.(16)

The limitations of our study include its retrospective nature. Not every patient diagnosed with Crohn's disease at our institution was included. Some patients did not undergo SBFT imaging within the allotted time frame required as part of our study design. 
Due to physician choice, often patients may not have undergone SBFT imaging at all. Also, SBFT studies are often interpreted real-time and retrospective review of images allowed only for our reinterpretation of static images. Some patients in the study may have been lost to follow up due to transition to a different pediatric gastroenterologist or an adult gastroenterologist.

Our study was also limited by the rapidly changing treatments available for Crohn’s disease during the time course of this study. Infliximab was approved by the FDA for Crohn's disease for adults in 1998 and for pediatrics in 2006, adalimumab was approved by the FDA for Crohn's disease for adults in 2007, and certolizumab was approved by the FDA for Crohn's disease in adults in 2008. Despite these approvals, the risks of surgery noted in this study are consistent with prior studies. In addition, the risks of surgery during the first year following diagnosis, especially in the cohort of patients with more abnormal small bowel imaging studies, may not be amenable to more aggressive therapy, as fibrostenotic changes may have occurred prior to diagnosis.

Although our study used SBFT to define small bowel radiologic abnormalities, MRE is rapidly becoming the routine radiological imaging of choice in pediatric Crohn's disease, due to its excellent cross-sectional findings and lack of radiation. A systematic review of MRE for assessment of small bowel activity in pediatric Crohn's disease gave excellent sensitivity and specificity, $84 \%$ and $97 \%$ respectively, using endoscopy as reference standard, although heterogeneity in bowel preparation, scanning technique, and reporting methodology was noted(17). A study of preoperative MRE in patients with Crohn's disease noted accuracy for identification of abscesses but not fistulas or stenoses(18), suggesting the need for careful intraoperative search for additional lesions. Nevertheless, MRE is becoming the imaging modality of choice in pediatric patients with Crohn's disease(19). 
In conclusion, current guidelines published by the European Society for Pediatric Gastroenterology, Hepatology and Nutrition (ESPGHAN) have radiological evaluation of the small bowel as a mandatory part of the evaluation of a patient suspected to have inflammatory bowel disease, except in cases where a definite diagnosis of ulcerative colitis is made(1). At this time, the North American Society for Pediatric Gastroenterology, Hepatology and Nutrition (NASPHGAN) has no current recommendations for imaging the small bowel in newly diagnosed Crohn's disease patients. We believe that all newly diagnosed patients with Crohn's disease would benefit from radiological imaging of the small bowel. This imaging may help counsel patients and families about long-term surgical risk and may help guide treatment.

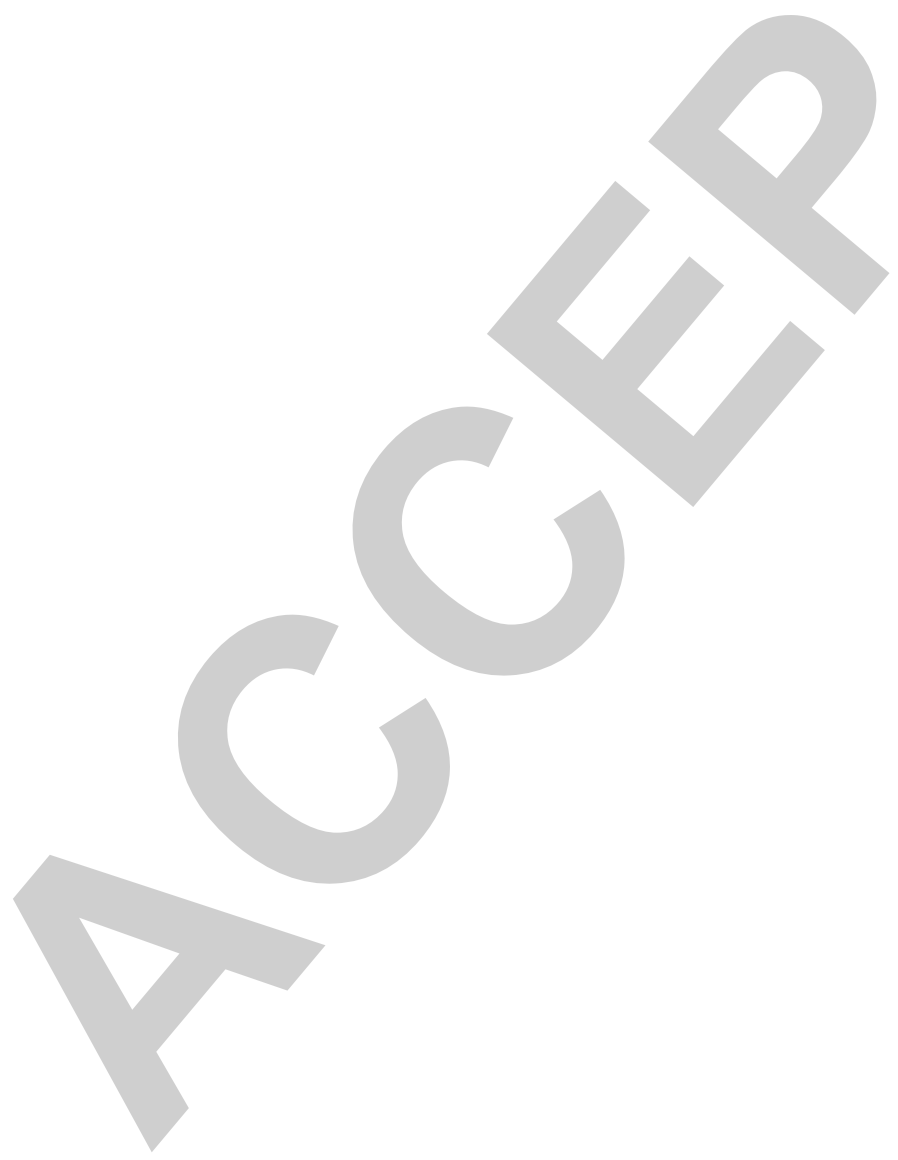




\section{References}

1 Ibd Working Group of the European Society for Paediatric Gastroenterology H, Nutrition Inflammatory bowel disease in children and adolescents: recommendations for diagnosis--the Porto criteria. Journal of Pediatric Gastroenterology and Nutrition 2005;41(1):1-7.

2 Al-Hawary M, Zimmermann EM A new look at Crohn's disease: novel imaging techniques. Curr Opin Gastroenterol 2012;28(4):334-40.

3 Cohen SA, Ephrath H, Lewis JD, et al. Pediatric capsule endoscopy: review of the small bowel and patency capsules. Journal of Pediatric Gastroenterology and Nutrition 2012;54(3):409-13.

4 de Ridder L, Mensink PB, Lequin MH, et al. Single-balloon enteroscopy, magnetic resonance enterography, and abdominal US useful for evaluation of small-bowel disease in children with (suspected) Crohn's disease. Gastrointestinal Endoscopy 2012;75(1):87-94.

5 Di Nardo G, Oliva S, Aloi M, et al. Usefulness of single-balloon enteroscopy in pediatric Crohn's disease. Gastrointestinal Endoscopy 2012;75(1):80-6.

6 Buchman AL, Miller FH, Wallin A, et al. Videocapsule endoscopy versus barium contrast studies for the diagnosis of Crohn's disease recurrence involving the small intestine. American Journal of Gastroenterology 2004;99(11):2171-7.

7 Schoepfer AM, Dehlavi MA, Fournier N, et al. Diagnostic delay in Crohn's disease is associated with a complicated disease course and increased operation rate. American Journal of Gastroenterology 2013;108(11):1744-53; quiz 54. 
8 Gupta N, Cohen SA, Bostrom AG, et al. Risk factors for initial surgery in pediatric patients with Crohn's disease. Gastroenterology 2006;130(4):1069-77.

9 Bernell O, Lapidus A, Hellers G Risk factors for surgery and postoperative recurrence in Crohn's disease. Annals of Surgery 2000;231(1):38-45.

10 Cerqueira RM, Lago PM Clinical factors predictive of Crohn's disease complications and surgery. European Journal of Gastroenterology and Hepatology 2013;25(2):129-

34.

11 Lacher M, Helmbrecht J, Schroepf S, et al. NOD2 mutations predict the risk for surgery in pediatric-onset Crohn's disease. Journal of Pediatric Surgery 2010;45(8):1591-7.

12 Nasir BF, Griffiths L, Nasir A, et al. Perianal disease combined with NOD2 genotype predicts need for IBD-related surgery in Crohn's disease patients from a populationbased cohort. Journal of Clinical Gastroenterology 2013;47(3):242-5.

13 Ryan JD, Silverberg MS, Xu W, et al. Predicting complicated Crohn's disease and surgery: phenotypes, genetics, serology and psychological characteristics of a population-based cohort. Alimentary Pharmacology and Therapeutics 2013;38(3):274-

83.

14 Ananthakrishnan AN, Gainer VS, Perez RG, et al. Psychiatric co-morbidity is associated with increased risk of surgery in Crohn's disease. Alimentary Pharmacology and Therapeutics 2013;37(4):445-54. 
15 Boualit M, Salleron J, Turck D, et al. Long-term outcome after first intestinal resection in pediatric-onset Crohn's disease: a population-based study. Inflammatory Bowel Diseases 2013;19(1):7-14.

16 Lazarev M, Huang C, Bitton A, et al. Relationship between proximal Crohn's disease location and disease behavior and surgery: a cross-sectional study of the IBD Genetics Consortium. American Journal of Gastroenterology 2013;108(1):106-12.

17 Giles E, Barclay AR, Chippington S, et al. Systematic review: MRI enterography for assessment of small bowel involvement in paediatric Crohn's disease. Alimentary Pharmacology and Therapeutics 2013;37(12):1121-31.

18 Seastedt KP, Trencheva K, Michelassi F, et al. Accuracy of CT enterography and magnetic resonance enterography imaging to detect lesions preoperatively in patients undergoing surgery for Crohn's disease. Diseases of the Colon and Rectum 2014;57(12):1364-70.

19 Maccioni F, Al Ansari N, Mazzamurro F, et al. Detection of Crohn disease lesions of the small and large bowel in pediatric patients: diagnostic value of MR enterography versus reference examinations. AJR. American Journal of Roentgenology 2014;203(5):W533-42. 


\section{Figure Legends}

Figure 1: Distribution of small bowel follow-through scores among the 232 patients included in the study. Grade 0 (normal), Grade 1 (minimal nodularity, ulcerations, normal luminal diameter, < 5cm involved), Grade 2 (more extensive ulcers, minimal luminal narrowing, 5-10 cm involved), Grade 3 (fistula, skip areas, extensive ulceration, >10 cm involved but no proximal bowel dilatation) and Grade 4 (proximal bowel dilatation).

Figure 2: Kaplan-Meier curve comparing patients in Group 0 (Grades 0-2 small bowel followthrough) with Group 1 (Grades 3-4 small bowel follow-through). Proportion surviving indicates percentage of patients who remained surgery-free. Time measured in days from diagnosis of Crohn's disease. 
Figure 1

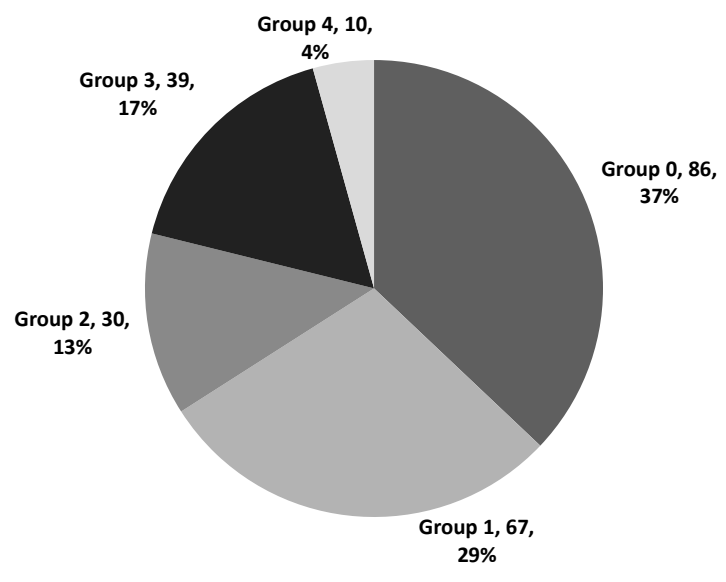

Copyright @ ESPGHAN and NASPGHAN. All rights reserved. 
Figure 2

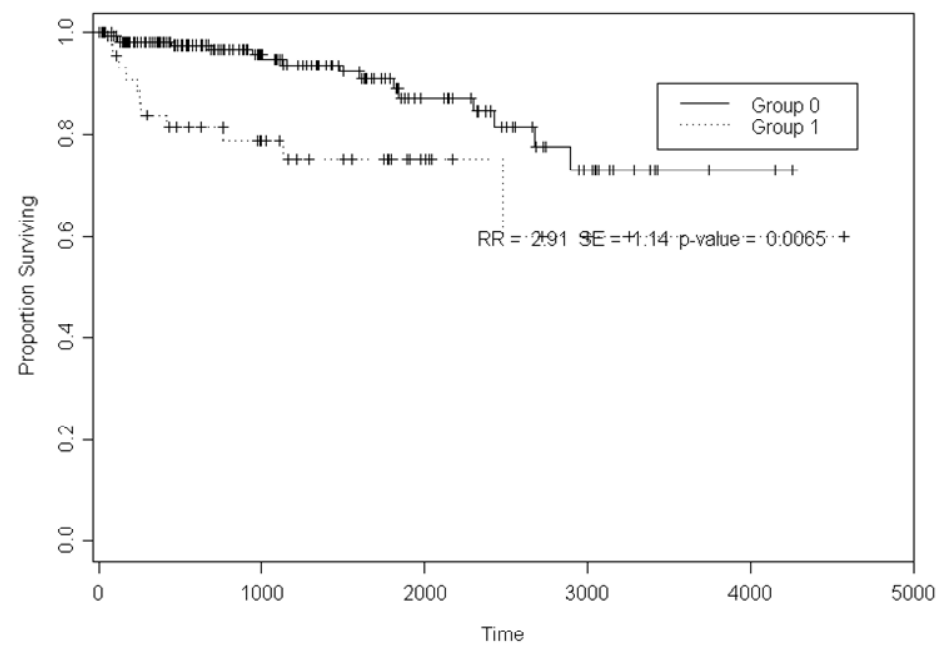

Copyright @ ESPGHAN and NASPGHAN. All rights reserved. 


\begin{tabular}{|lcccc|}
\hline $\begin{array}{l}\text { TABLE 1. Frequency of small bowel surgical intervention in newly diagnosed pediatric Crohn's disease } \\
\text { patients. }\end{array}$ \\
\hline Group & $\mathrm{n}$ & 1 year after diagnosis & 5 years after diagnosis & 8 years after diagnosis \\
\hline All patients & 232 & $5 \%$ & $16 \%$ & $33 \%$ \\
\hline Grade 0-2 imaging & 183 & $2 \%$ & $11 \%$ & $29 \%$ \\
\hline Grade 3-4 imaging & 49 & $17 \%$ & $27 \%$ & $42 \%$ \\
& & & & \\
\end{tabular}

